\title{
A knowledge translation perspective on executive function in clinical practice to mitigate chronic pain
}

\author{
Zakir Uddin, Joy C. MacDemid, and Victoria Galea
}

\section{McMaster University}

\section{Executive Function}

Executive Function (EF) is considered the controlling mechanism of cognition dynamics; ${ }^{1}$ where the term "cognition" refers to all neural processes by which sensory information is transformed, modulated (reduced or elaborated), preserved (stored or recovered), and used. ${ }^{2}$ To realize the biological process of cognition, we have to consider the information process in neural networks beyond the individual neuron. ${ }^{3} \mathrm{EF}$ acts as a dynamic part of cognition in the conscious control of thought. From an evolutionary biology perspective, EF is an adaptive response and plays a major role in self-directed sensorymotor action. ${ }^{4}$ One may think of it as a collection of interrelated processes that control and regulate thought and action, and that are capable of suppressing habitual responses. EF enables the ability to attend selectively to a stimulus and to inhibit the distraction of other stimuli. A combined analysis demonstrated that the lateral prefrontal cortex (LPFC) cascades EF from premotor to anterior PFC acting as a controlling mechanism depending on the stimuli, context, and episode. ${ }^{5}$ The PFC neurons work as part of an integrative network of $\mathrm{EF}$ and control cognition with the optimum motor response (Figure 1). A confirmatory factor analysis study ${ }^{1}$ suggested a taxonomy of EF comprised of three moderately correlated, but separable, facets: shifting (task switching), updating (working memory), and inhibition (behavioral inhibition).

EF is conceptualized ${ }^{1}$ as the capability of action selection in relation to internal goals organized by the Prefrontal Cortex (PFC). The PFC is functionally important for the temporal integration of sensory information (via the multimodal sensory cortex) in the sequence of optimum behaviour according to the internal goal. ${ }^{3,6,7}$ In the PFC, EF operates through dynamic cross-modal association with a temporal organization of behaviour according to the transferred information, like a perception-action cycle. ${ }^{7}$ In that cycle, the multimodal sensory association area is integrating sensory information towards movement planning and thinking as an anatomical substrate of higher brain function (e.g. conscious thought, perception, and goal-directed action). ${ }^{3}$ The PFC is the highest level of the physiological cortical hierarchy and assumes the prime role for representation and execution of actions. ${ }^{7}$ To sum up, cognitive control (i.e. EF) is accomplished by framing of temporal action and by the selection of events, in other words contextual versus episodic control of communication (Figure 1).

\section{Potential Role of EF in Mitigate Chronic Pain}

In view of pain neurophysiology, subjective interpretation of pain message goes to three specialized regions of the brain: 1) The Somatosensory Cortex (physical sensation region), 2) The Limbic System (emotional feeling region), and 3) The Frontal Cortex (the thinking or cognitive

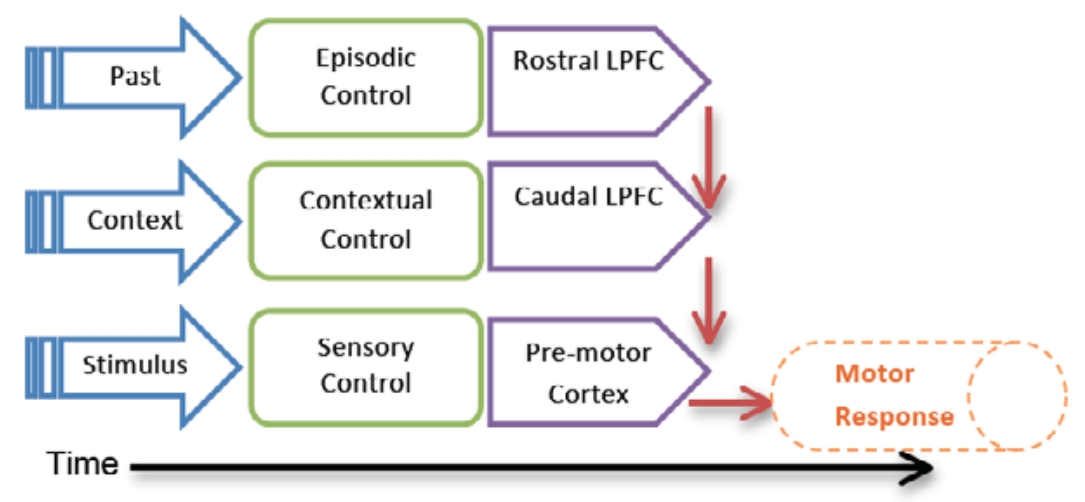

Figure 1: Temporal functional cognitive control processing model (adapted from Koechlin E, et al. 2003, 2007). ${ }^{5,6}$ LPFC = Lateral Prefrontal Cortex. 
region). Thefrontal cortexisconsidered the most responsible area for pain generation, maintenance, integration, and control. More specifically, the PFC is considered as a key source of pain modulation. ${ }^{8}$ Evidence suggests that the PFC actively reorganizes after chronic exposure to pain. ${ }^{9,10}$ Brain imaging studies demonstrated parallel activation in areas of the brain for $E F$ and the experience of chronic pain. Also, people with chronic pain suffer from poor executive ability ${ }^{11,12}$ presumably because they do not have sufficient access to EF to mitigate their chronic pain experiences. Therefore, rehabilitation strategies should aim to improve EF by targeting the areas of the brain that share function with chronic pain.

The top-down pain modulation (pain

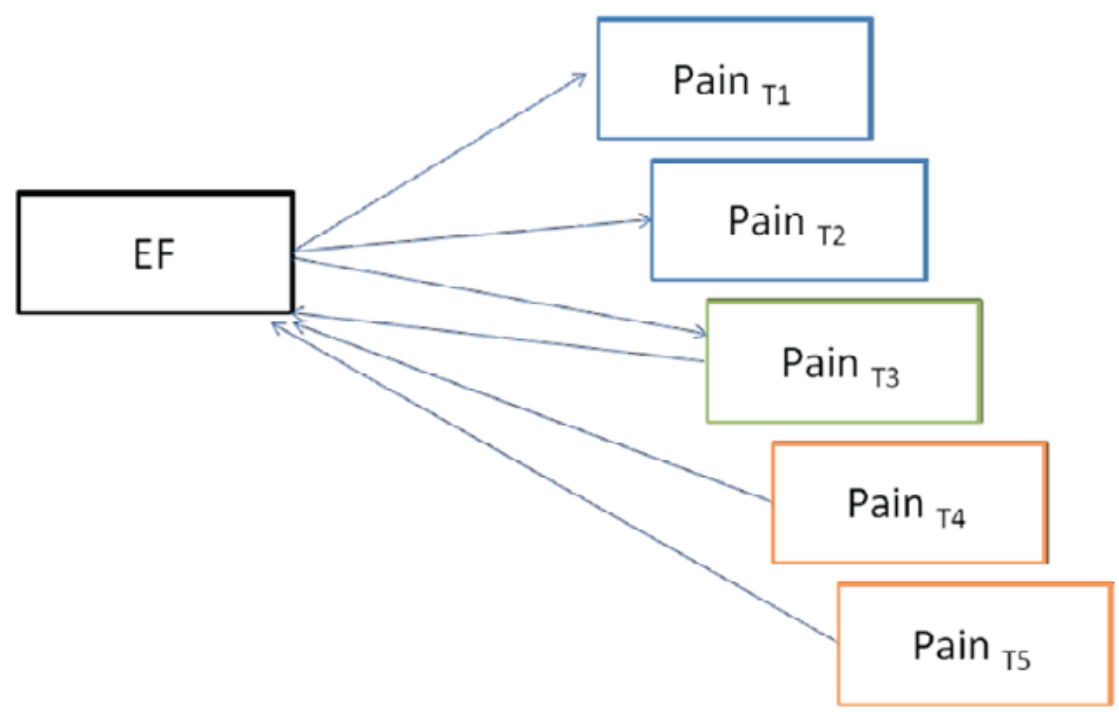

Figure 2: A reciprocal dynamic relationship between EF and Pain, ${ }^{15}$ where both are engaged in the overlapping function and capacity-limited cognitive resources over the time (T1 and $\mathrm{T} 2=$ acute stage, $\mathrm{T} 3=$ transition stage, $\mathrm{T} 4$ and $\mathrm{T} 5=$ chronic stage). In the chronic stage, pain is inversely related to executive function..$^{11,12}$ inhibitory mechanism) is maintained by a cognitive control mechanism, ${ }^{13}$ either by integrating homeostatic regulation or autonomic premotor area activation. Rationally, the faulty top-down control is considered to explain the development of chronic pain. ${ }^{14}$ Within top-down cognitive control, pain and EF (i.e. cognitive control mechanism) can be seen as an interlinked dynamic phenomenon in different stages of the pain process, especially in the acute to chronic stage (Figure 2). The pain flare-up condition might further sensitize the nervous system. Appropriate use of EF interventions might help to desensitize the system through the operant conditioning (active behaviour) mechanisms.

Movement and function limitations are common in chronic pain, and it is linked with pain related disability. EF linking strategy (e.g. pacing and graded exposure) might be helpful to reduce pain related disability by maximizing movement and function. Therefore, treatment approaches focusing on cognition improvement (e.g. graded motor imagery, goal setting, altering visual input, graded exposure, tone-pitch recognition, sensory discrimination training programmes, desensitization approach, and sensory-motor interaction targeted exercise) might improve EF and as well as the movement pattern in chronic pain.

\section{Conclusion}

Applying EF to the management of pain in a clinical setting can enact a potential source of pain modulation. ${ }^{8}$ The conscious control of thought and action are fundamental to motor rehabilitation in chronic pain. Because of the manipulating power in cross-temporal contingencies, EF is important in healthy behaviour practice as well as in the clinical practice of pain management. Further research and effective knowledge translation are essential in this area of research and practice.

\section{References}

1. Miyake A, Friedman NP, Emerson MJ, Witzki AH, Howerter A, Wager TD. The unity and diversity of executive functions and their contributions to complex "Frontal Lobe" tasks: a latent variable analysis. Cognit Psychol. 2000;41(1):49-100.

2. Neisser U. Cognitive psychology. New York: Appleton-Century-Crofts; 1967.

3. Kandel ER. From nerve cells to cognition: The internal cellular representation required for perception and action. Principles of neural science. 2000;4:381-408.

4. Barkley RA. The executive functions and self-regulation: An evolutionary neuropsychological perspective. Neuropsychol Rev. 2001;11(1):1-29.

5. Koechlin E, Ody C, Kouneiher F. The architecture of cognitive control in the human prefrontal cortex. Science. 2003;302(5648):1181. 
6. Koechlin E, Summerfield C. An information theoretical approach to prefrontal executive function. Trends Cogn Sci (Regul Ed ). 2007;11(6):229-235.

7. Fuster JM. The prefrontal cortex. London: Academic Press; 2008.

8. Bingel U, Tracey I. Imaging CNS modulation of pain in humans. Physiology. 2008;23(6):371-380.

9. Wager TD, Rilling JK, Smith EE, et al. Placebo-induced changes in FMRI in the anticipation and experience of pain. Science. 2004;303(5661):1162.

10. Baliki MN, Schnitzer TJ, Bauer WR, Apkarian AV. Brain morphological signatures for chronic pain. PloS one. 2011;6(10): 26010.

11. Oosterman J, Derksen LC, van Wijck AJ, Kessels RP, Veldhuijzen DS. Executive and attentional functions in chronic pain: Does performance decrease with increasing task load? Pain Res Manag. 2012;17(3):159-165.

12. Abeare CA, Cohen JL, Axelrod BN, Leisen JCC, Mosley-Williams A, Lumley MA. Pain, executive functioning, and affect in patients with rheumatoid arthritis. Clin J Pain. 2010;26(8):683.

13. Tracey I, Mantyh PW. The cerebral signature for pain perception and its modulation. Neuron. 2007;55(3):377-391.

14. Ossipov MH, Dussor GO, Porreca F. Central modulation of pain. J Clin Invest. 2010;120(11):3779.

15. Buhle J, Wager TD. Performance-dependent inhibition of pain by an executive working memory task. Pain. 2010;149(1):19-26.

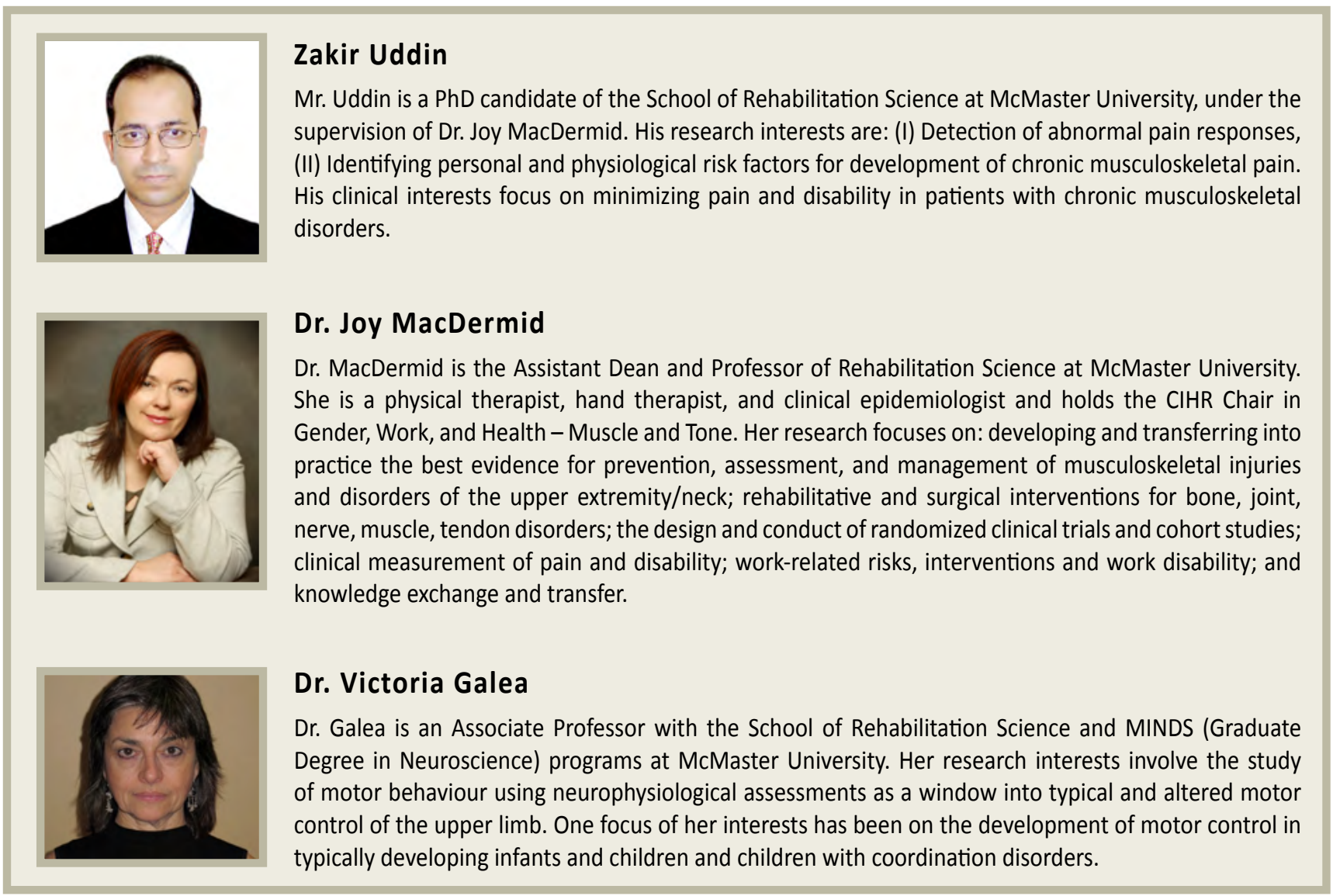

